\title{
Antimicrobial, $\alpha$-Glucosidase and Alkaline Phosphatase Inhibitory Ac- tivities of Bergenin, The Major Constituent of Cissus populnea Roots
}

\author{
Jean Noël Nyemb ${ }^{1 *}$, Madeleine T Djankou1, Emmanuel Talla², Alembert T Tchinda ${ }^{3}$, David T Ngoudjou ${ }^{4}$, Jamshed Iqbal ${ }^{5}$ and Joseph T Mbafor \\ ${ }^{1}$ Department of Organic Chemistry, Faculty of Science, University of Yaounde I, PO Box 812, Yaounde, Came-roon \\ ${ }^{2}$ Department of Chemistry, Faculty of Science, University of Ngaoundere, PO Box 454, Ngaoundere, Cameroon \\ ${ }^{3}$ Institute of Medical Research and Medicinal Plants Studies (IMPM), Ministry of Scientific Research and Inno-vation, PO Box 6163 Yaounde, Cameroon \\ ${ }^{4}$ Department of Biochemistry, Faculty of Science, University of Dschang, PO Box 67, Dschang, Cameroon \\ ${ }^{5}$ Centre for Advanced Drug Research, COMSATS Institute of Information Technology, Abbottabad-22060, Pakistan
}

\begin{abstract}
Bergenin (1) was isolated as the major compound of the stem barks of Cissus populnea. Its structure was elucidated on the basis of spectral analysis, including 1D and 2D NMR experiments and Mass Spectrometry (MS) as well as by comparison with available data in the literature. Bergenin (1) was evaluated for its antimicrobial activity against four bacteria strains (Salmonella typhi ATCC6539, Salmonella typhi (isolate), Pseudomonas aeruginosa ATCC9721 and Escherichia coli (isolate)) and four yeasts (Candida albicans ATCC9002, Candida parapsilosis ATCC22019, Candida krusei (isolate) and Candida albicans (isolate)) using micro broth dilution method and for its enzymatic activities against $\alpha$-glucosidase and Alkaline Phosphatase ( $h$-TNAP and $h$-IAP). It exhibited significant to moderate antibacterial activities with the MIC ranking from 8 to $64 \mu \mathrm{g} \cdot \mathrm{mL}^{-1}$, while it appeared to be inactive (MIC>125 $\mu \mathrm{g} \cdot \mathrm{mL}^{-1}$ ) against all the tested yeast. However, it was found to be high potent inhibitor of both $h$-TNAP and $h$-IAP alkaline phosphatase isoenzymes, with a selectivity for the $h$-TNAP. Daucosterol (4) and a mixture of sitosterol $(\mathbf{2 + 3})$ were also obtained and were screening for the same bioactivities.
\end{abstract}

Keywords: Cissus populnea; Bergenin; Antibacterial; a-Glucosidase; Alkaline phosphatase

\section{Introduction}

Cissus populnea belongs to the Vitaceae family, a grape family of angiosperm with about 14 genera and 900 species principally distributed in tropical regions, with a few genera in temperate regions [1]. C. populnea is very little known in Cameroon and not well documented. However, it is an important medicinal plant in Nigeria where it is very famous for its uses in traditional medicine as well as like a spice. The plant is cited in the treatment of several infectious diseases including male infertility, urinary infections, skin diseases, indigestion, wounds, venereal diseases, gonorrhoea, ulcers, jaundice, icterus, trypanosomiasis [2]. C. populnea is also used against inflammations, colics, hypertension, hemorrhoids, fever, oedema, yellow fever, leprosy $[3,4]$, malaria [5]. The roots of the plant are used in Adamawa region of Cameroon for the treatment of breast cancer [6]. C. populnea has been extensively studied biologically. Antimalarial activity [5,7], larvicidal activity [8], antimicrobial properties [9,10], antianaemic activities [11], spermatogenic potentials [12], hypoglycemic activity [13], as well as antiproliferative activities $[6,14]$ of the plant parts have been reported. Previous qualitative phytochemical screening revealed that the aqueous extract of the stem bark contains flavonoids, steroids, saponins and tanins [12]. The stem barks were also found to contain carbohydrates, tannins, cyanogenic glycosides, saponins and anthraquinones $[15,16]$. The quantitative phytochemical analysis of the plant has also been reported $[3,4]$. However, to the best of our knowledge, no studies related to the isolation of compounds from this plant have been reported.

In the present investigation, the extraction and the isolation of the roots of C. populnea gave a brown extract from which Bergenin (1) was isolated. Bergenin (1) is a crystalline compound which has been previously isolated from Cissus pteroclada $[17,18]$ and from several other plant species [19-23]. However, this paper reports for the first time its isolation from $C$. populnea. Furthermore, extraction of the roots of C. populnea gave in addition to Bergenin (1) a mixture of sitosterol (2 and 3), and Daucosterol (4). The isolated compounds were evaluated for their antimicrobial, $\alpha$-glucosidase, and alkaline phosphatase inhibitory activities.

Alkaline Phosphatases (APs) are enzymes present in large quantities in the liver and bones. These enzymes can be used as markers in certain cancers, since an increase in their level may be related to bone cancer, liver cancer or others. However, this over expression of APs has been reported in patients with type 2 Diabetes for several years [24]. The use of a-glucosidase inhibitors is considered to be an effective strategy in the treatment of diabetes. Several $\alpha$-glucosidase inhibitors, such as acarbose and voglibose obtained from natural sources have been used clinically in the treatment of diabetes mellitus [25], since they control effectively the blood glucose level after food intake. Unfortunately, only a few $\alpha$-glucosidase inhibitors are commercially available and most of them are often associated with a number of serious side effects [26]. Screening of $a$-glucosidase and APs inhibitors from plants and synthetic sources is increasing. Inhibitors of these enzymes have been recently developed from natural sources $[27,28]$.

\section{Experimental Protocols}

\section{General experimental procedures}

Column chromatography (CC) was performed on silica gel 60 (Merck, 63-200 $\mu \mathrm{m}$ ) with a gradient of n-hexane-EtOAc then, EtOAc$\mathrm{MeOH}$ for the elution. For analytical Thin Layer Chromatography

*Corresponding author: Jean Noël Nyemb, Department of Organic Chemistry, University of Yaounde 1, PO Box 812 Yaounde, Cameroon, Tel: +237674759584 E-mail: nyembjeannoel@gmail.com

Received February 01, 2018; Accepted February 05, 2018; Published February 12, 2018

Citation: Nyemb JN, Djankou MT, Talla E, Tchinda AT, Ngoudjou DT, et al. (2018) Antimicrobial, $\boldsymbol{\alpha}$-Glucosidase and Alkaline Phosphatase Inhibitory Activities of Bergenin, The Major Constituent of Cissus populnea Roots. Med Chem (Los Angeles) 8: 021-025. doi: 10.4172/2161-0444.1000492

Copyright: (c) 2018 Nyemb JN, et al. This is an open-access article distributed under the terms of the Creative Commons Attribution License, which permits unrestricted use, distribution, and reproduction in any medium, provided the original author and source are credited. 
Citation: Nyemb JN, Djankou MT, Talla E, Tchinda AT, Ngoudjou DT, et al. (2018) Antimicrobial, a-Glucosidase and Alkaline Phosphatase Inhibitory Activities of Bergenin, The Major Constituent of Cissus populnea Roots. Med Chem (Los Angeles) 8: 021-025. doi: 10.4172/21610444.1000492

(TLC), silica gel precoated aluminium sheets $\mathrm{F}_{254}$ Merck $(20 \times 20$ $\mathrm{cm})$ were used. The TLC plates after development were visualized under UV light ( 254 and $365 \mathrm{~nm}$ ) and then sprayed with $15 \%$ sulfuric acid followed by heating at $105^{\circ} \mathrm{C}$ for $15 \mathrm{~min}$. Melting points of the compounds were measured in open capillaries using Stuart melting point apparatus (SMP-3) and are uncorrected. ${ }^{1} \mathrm{H}(500 \mathrm{MHz})$ and ${ }^{13} \mathrm{C}$ (125 MHz) NMR spectra were recorded on a Bruker Avance AV-500 spectrometer, with chemical shifts given in $\mathrm{ppm}$. The spectra were run using $\mathrm{CDCl}_{3}$ or DMSO- $d_{6}$ as solvents and TMS as internal standard. ESI-MS spectra were measured on a Q-TOF Ultima spectrometer (Waters). Ciprofloxacin (Sigma-Aldrich, St Quentin Fallavier, France), and Ketoconazole (European Pharmacopoeia, Sigma-Aldrich) were used as reference standards respectively for antibacterial and antifungal assay. All organic solvents used for the tests were upgrade. Water used was distilled. All solutions were used on the day of preparation [29-31].

\section{Plant material}

The roots of C. populnea Guill \& Perr were collected in October 2015 in the Soudano-Guinea savanna of the University of Ngaoundere, Region of Adamawa, Cameroon. The samples were taxonomically identified by $M$. Nana Victor, botanist at the National Herbarium of Yaounde (Cameroon) where a voucher specimen $(36962 / \mathrm{HNC})$ is deposited.

\section{Extraction and isolation}

Air-dried powder of roots $(300 \mathrm{~g})$ were extracted by maceration $(2 \mathrm{~L})$ resulting after evaporation to dryness under reduced pressure to a dark brown extract $(20.72 \mathrm{~g})$ which was then refluxed in EtOAc $(750 \mathrm{~mL})$ three rounds of four hours each. All the EtOAc extracts were combined and then evaporated to dryness under reduced pressure at $40^{\circ} \mathrm{C}$ to a minimum volume and allowed to stand resulting in the precipitation of a white solid. This precipitate was washed several times using hexane, EtOAc, and acetone then recrystallized in $\mathrm{MeOH}$. After recrystallization, the $\mathrm{MeOH}$ soluble portion was passed through a column chromatography of sephadex LH-20 using a mixture of EtOAc/MeOH 8:2 as solvent of elution leading to compound $\mathbf{1}$ as a white powder $(510 \mathrm{mg})$. The remaining EtOAc fraction $(7.8 \mathrm{~g})$ was chromatographed on a silica gel column and eluted with a gradient of increasing polarity using Hexane/EtOAc (1:0-0:1, v/v) and EtOAc/ $\mathrm{MeOH}(1: 0-0: 1, \mathrm{v} / \mathrm{v})$ as solvent. From the main column, in addition of $\mathbf{1}$, compounds $\mathbf{2}$ and $\mathbf{3}$ were obtained as a mixture in a ratio (2.4:1) and compounds 4 as a pure compound.

Bergenin (1): white powder, m.p. $157-159^{\circ} \mathrm{C}$; Rf 0.45 [EtOAc: $\mathrm{MeOH}(9: 1)]$; TOF-MS-ESI+ m/z $351.1[\mathrm{M}+\mathrm{Na}]^{+}$for $\mathrm{C}_{14} \mathrm{H}_{16} \mathrm{O}_{9} \cdot{ }^{1} \mathrm{H}$ NMR $\left(\mathrm{CDCl}_{3}\right), \delta: 7.00(1 \mathrm{H}, s, \mathrm{H}-6), 9.77(1 \mathrm{H}, s, \mathrm{COOH}), 8.46(1 \mathrm{H}, s$, $\mathrm{OH}-4), 3.78(6 \mathrm{H}, s, \mathrm{OCH} 3-5), 3.39(3 \mathrm{H}, s, 3-\mathrm{OCH} 3), 5.00(1 \mathrm{H}, d, \mathrm{~J}=10.5$ $\left.\mathrm{Hz}, \mathrm{H}-1^{\prime}\right), 4.00\left(1 \mathrm{H}, d d, \mathrm{~J}=10.5 ; 9.5 \mathrm{~Hz}, \mathrm{H}-2^{\prime}\right), 3.66(1 \mathrm{H}, t d, \mathrm{~J}=9.1 ; 5.4$ $\left.\mathrm{Hz}, \mathrm{H}-3^{\prime}\right), 3.20\left(1 \mathrm{H}, t d, \mathrm{~J}=9.3\right.$; $\left.5.4 \mathrm{~Hz}, \mathrm{H}-4^{\prime}\right), 3.58(1 \mathrm{H}, d d d, \mathrm{~J}=9.7 ; 7.7$; $\left.2.1 \mathrm{~Hz}, \mathrm{H}-5^{\prime}\right), 3.45\left(1 \mathrm{H}, d d, \mathrm{~J}=13.1 ; 5.6 \mathrm{~Hz}, \mathrm{H}-6 \mathrm{a}^{\prime}\right), 3.85(1 \mathrm{H}, d, \mathrm{~J}=11.6$ $\left.\mathrm{Hz}, \mathrm{H}-6 \mathrm{~b}^{\prime}\right), 3.38\left(2 \mathrm{H}, o v, \mathrm{OH}-2^{\prime}\right), 5.69\left(1 \mathrm{H}, d, \mathrm{~J}=5.4 \mathrm{~Hz}, \mathrm{OH}-3^{\prime}\right), 5.47$ $\left(1 \mathrm{H}, d, \mathrm{~J}=5.4 \mathrm{~Hz}, \mathrm{OH}-4^{\prime}\right), 4.94\left(1 \mathrm{H}, t l, \mathrm{~J}=10.5 \mathrm{~Hz}, \mathrm{OH}-6^{\prime}\right) .{ }^{13} \mathrm{C}$ NMR $\left(125 \mathrm{MHz}, \mathrm{CDCl}_{3}\right) \delta: 118.0$ (C, C-1), $115.9(\mathrm{CH}, \mathrm{C}-2), 148.0$ (C, C-3), 150.8 (C, C-4), 140.5 (C, C-5), 109.4 (C, C-6), 72.0 (CH, C-1'), 79.7 $\left(\mathrm{CH}, \mathrm{C}-2^{\prime}\right), 73.9\left(\mathrm{CH}, \mathrm{C}-3^{\prime}\right), 70.9\left(\mathrm{CH}, \mathrm{C}-4^{\prime}\right), 81.9\left(\mathrm{CH}, \mathrm{C}-5^{\prime}\right), 61.0$ $\left(\mathrm{CH} 2, \mathrm{C}-6^{\prime}\right), 163.3$ (C, CO) (Figure 1).

$\boldsymbol{\beta}$-sitosterol (2): colourless needles, Rf 0.35 [Hexane/AcOEt (9:1)]; ${ }^{1} \mathrm{H}-\mathrm{NMR}\left(\mathrm{CDCl}_{3}\right), \delta: 5.35(1 \mathrm{H}, d, \mathrm{~J}=5.1 \mathrm{~Hz}, \mathrm{H}-6), 3.53(1 \mathrm{H}, d d d, \mathrm{~J}=15.9$, 11.0, $4.6 \mathrm{~Hz}, \mathrm{H}-3), 1.04(3 \mathrm{H}, s, \mathrm{H}-19), 1.01\left(3 \mathrm{H}, d, \mathrm{~J}=7.0 \mathrm{~Hz}, \mathrm{H}_{3}-27\right)$, $0.95\left(3 \mathrm{H}, d, J=6.6 \mathrm{~Hz}, \mathrm{H}_{3}-26\right), 0.88\left(3 \mathrm{H}, \mathrm{d}, J=1.8 \mathrm{~Hz}, \mathrm{H}_{3}-21\right), 0.85(3 \mathrm{H}$, ov, $\left.\mathrm{H}_{3}-29\right)$ and $0.71\left(3 \mathrm{H}, \mathrm{s}, \mathrm{H}_{3}-18\right) .{ }^{13} \mathrm{C}-\mathrm{NMR}\left(\mathrm{CDCl}_{3}\right): \delta 37.3\left(\mathrm{CH}_{2}\right.$, $\mathrm{C}-1), 31.9\left(\mathrm{CH}_{2}, \mathrm{C}-2\right), 71.8(\mathrm{CH}, \mathrm{C}-3), 42.4\left(\mathrm{CH}_{2}, \mathrm{C}-4\right), 140.8(\mathrm{C}-5)$, 121.7 (CH, C-6), $31.7\left(\mathrm{CH}_{2}, \mathrm{C}-7\right), 31.9(\mathrm{CH}, \mathrm{C}-8), 50.2(\mathrm{CH}, \mathrm{C}-9), 36.6$ (C, C-10), $21.1\left(\mathrm{CH}_{2}, \mathrm{C}-11\right), 39.8\left(\mathrm{CH}_{2}, \mathrm{C}-12\right), 42.3(\mathrm{CH}, \mathrm{C}-13), 56.8$ (CH, C-14), $24.3\left(\mathrm{CH}_{2}, \mathrm{C}-15\right), 28.2\left(\mathrm{CH}_{2}, \mathrm{C}-16\right), 56.1(\mathrm{CH}, \mathrm{C}-17), 11.9$ $\left(\mathrm{CH}_{3}, \mathrm{C}-18\right), 19.0\left(\mathrm{CH}_{3} \mathrm{C}-19\right), 36.2\left(\mathrm{CH}_{2}, \mathrm{C}-20\right), 18.8\left(\mathrm{CH}_{3}, \mathrm{C}-21\right), 34.0$ $\left(\mathrm{CH}_{2}, \mathrm{C}-22\right), 26.3\left(\mathrm{CH}_{2}, \mathrm{C}-23\right), 45.9(\mathrm{CH}, \mathrm{C}-24), 29.3(\mathrm{CH}, \mathrm{C}-25), 19.4$ $\left(\mathrm{CH}_{3}, \mathrm{C}-26\right), 19.8\left(\mathrm{CH}_{3}, \mathrm{C}-27\right), 23.1\left(\mathrm{CH}_{2}, \mathrm{C}-28\right), 12.0\left(\mathrm{CH}_{3}, \mathrm{C}-29\right)$.

Stigmasterol (3): colourless needles, Rf 0.35 [Hexane/AcOEt (9:1)]; ${ }^{1} \mathrm{H}$ NMR $\left(\mathrm{CDCl}_{3}\right), \delta: 5.38(1 \mathrm{H}, \mathrm{m}, \mathrm{H}-6), 5.14(1 \mathrm{H}, o v, \mathrm{H}-22), 5.05(1 \mathrm{H}$, $d d, \mathrm{~J}=15.2,8.7 \mathrm{~Hz}, \mathrm{H}-23), 3.56(1 \mathrm{H}, d d d, \mathrm{~J}=15.8,11.0,4.5 \mathrm{~Hz}, \mathrm{H}-3), 1.04$ (3H, s, H-19), $1.01\left(3 \mathrm{H}, \mathrm{d}, \mathrm{J}=7.0 \mathrm{~Hz}, \mathrm{H}_{3}-27\right), 0.95\left(3 \mathrm{H}, \mathrm{d}, J=6.6 \mathrm{~Hz}, \mathrm{H}_{3}-\right.$ 26), $0.88\left(3 \mathrm{H}, \mathrm{d}, J=1.8 \mathrm{~Hz}, \mathrm{H}_{3}-21\right), 0.85\left(3 \mathrm{H}, o v, \mathrm{H}_{3}-29\right)$ and $0.71(3 \mathrm{H}$, s, $\left.\mathrm{H}_{3}-18\right) .{ }^{13} \mathrm{C} \mathrm{NMR}\left(126 \mathrm{MHz}, \mathrm{CDCl}_{3}\right) \delta: 37.2\left(\mathrm{CH}_{2}, \mathrm{C}-1\right), 31.9\left(\mathrm{CH}_{2}\right.$, C-2), $71.8(\mathrm{CH}, \mathrm{C}-3), 42.3\left(\mathrm{CH}_{2}, \mathrm{C}-4\right), 140.8$ (C, C-5), $121.7(\mathrm{CH}, \mathrm{C}-6)$, $31.7\left(\mathrm{CH}_{2}, \mathrm{C}-7\right), 31.9(\mathrm{CH}, \mathrm{C}-8), 50.2(\mathrm{CH}, \mathrm{C}-9), 36.5$ (C, C-10), 21.1 $\left(\mathrm{CH}_{2}, \mathrm{C}-11\right), 39.8\left(\mathrm{CH}_{2}, \mathrm{C}-12\right), 42.3(\mathrm{C}, \mathrm{C}-13), 56.8(\mathrm{CH}, \mathrm{C}-14), 24.3$ $\left(\mathrm{CH}_{2}, \mathrm{C}-15\right), 28.2\left(\mathrm{CH}_{2}, \mathrm{C}-16\right), 56.1(\mathrm{CH}, \mathrm{C}-17), 12.1\left(\mathrm{CH}_{3}, \mathrm{C}-18\right), 19.0$ $\left(\mathrm{CH}_{3}, \mathrm{C}-19\right), 40.5\left(\mathrm{CH}_{2}, \mathrm{C}-20\right), 18.8\left(\mathrm{CH}_{3}, \mathrm{C}-21\right), 138.4(\mathrm{CH}, \mathrm{C}-22)$, $129.3(\mathrm{CH}, \mathrm{C}-23), 51.2(\mathrm{CH}, \mathrm{C}-24), 45.9(\mathrm{CH}, \mathrm{C}-25), 19.4\left(\mathrm{CH}_{3}, \mathrm{C}-26\right)$, $19.8\left(\mathrm{CH}_{3}, \mathrm{C}-27\right), 24.3\left(\mathrm{CH}_{2}, \mathrm{C}-28\right), 12.3\left(\mathrm{CH}_{3}, \mathrm{C}-29\right)$.

Daucosterol (4): $\mathrm{C}_{35} \mathrm{H}_{60} \mathrm{O}_{6}$, colourless needles; TOF-MS-ESI+ m/z $599.2[\mathrm{M}+\mathrm{Na}]^{+}$for $\mathrm{C}_{35} \mathrm{H}_{60} \mathrm{O}_{6} \cdot{ }^{6} \mathrm{H}-\mathrm{NMR}$ (DMSO-d6): $\delta: 5.33(1 \mathrm{H}, m$, $\mathrm{H}-6), 4.22\left(1 \mathrm{H}, d, \mathrm{~J}=7.8 \mathrm{~Hz}, \mathrm{H}-1^{\prime}\right), 3.64(1 \mathrm{H}, d, \mathrm{~J}=11.1 \mathrm{~Hz}, \mathrm{H}-6 \mathrm{a}), 3.40$ $\left(1 \mathrm{H}, d d, \mathrm{~J}=11.1,5.4 \mathrm{~Hz}, \mathrm{H}-6^{\prime} \mathrm{b}\right), 3.02\left(1 \mathrm{H}, t, \mathrm{~J}=8.9 \mathrm{~Hz}, \mathrm{H}-4^{\prime}\right), 3.12(1 \mathrm{H}$, $\left.t, \mathrm{~J}=8.8 \mathrm{~Hz}, \mathrm{H}-3^{\prime}\right), 2.90\left(1 \mathrm{H}, t, \mathrm{~J}=8.3 \mathrm{~Hz}, \mathrm{H}-2^{\prime}\right), 3.07(1 \mathrm{H}, d d d, \mathrm{~J}=11.1$, 5.8, $\left.1.9 \mathrm{~Hz} \mathrm{H}-5^{\prime}\right), 3.46(1 \mathrm{H}, \mathrm{m}, \mathrm{H}-3), 2.11(1 \mathrm{H}, \mathrm{m}, \mathrm{H}-4 \mathrm{~b}), 2.35(1 \mathrm{H}, \mathrm{m}$, $\mathrm{H}-4 \mathrm{a}), 0.96\left(3 \mathrm{H}, \mathrm{s}, \mathrm{CH}_{3}-19\right), 0.90\left(3 \mathrm{H}, d, \mathrm{~J}=6.5 \mathrm{~Hz}, \mathrm{CH}_{3}-21\right), 0.82(3 \mathrm{H}$, $\left.m, \mathrm{~J}=7.2 \mathrm{~Hz}, \mathrm{CH}_{3}-29\right), 0.81\left(3 \mathrm{H}, d, \mathrm{~J}=6.9 \mathrm{~Hz}, \mathrm{CH}_{3}-26\right), 0.80(3 \mathrm{H}, d$, $\left.\mathrm{J}=6.9 \mathrm{~Hz}, \mathrm{CH}_{3}-27\right), 0.65\left(3 \mathrm{H}, s, \mathrm{CH}_{3}-18\right) ;{ }^{13} \mathrm{C}-\mathrm{NMR}\left(\mathrm{DMSO}-d_{6}\right): \delta: 36.8$ $\left(\mathrm{CH}_{2}, \mathrm{C}-1\right), 31.3\left(\mathrm{CH}_{2}, \mathrm{C}-2\right), 76.9(\mathrm{CH}, \mathrm{C}-3), 38.2\left(\mathrm{CH}_{2}, \mathrm{C}-4\right), 140.4(\mathrm{C}$, C-5), $121.2(\mathrm{CH}, \mathrm{C}-6), 31.4\left(\mathrm{CH}_{2}, \mathrm{C}-7\right), 31.3(\mathrm{CH}, \mathrm{C}-8), 49.6(\mathrm{CH}, \mathrm{C}-9)$, 35.5 (C, C-10), $20.6\left(\mathrm{CH}_{2}, \mathrm{C}-11\right), 39.2\left(\mathrm{CH}_{2}, \mathrm{C}-12\right), 41.8$ (C, C-13), 56.1 (CH, C-14), $23.8\left(\mathrm{CH}_{2}, \mathrm{C}-15\right), 27.8\left(\mathrm{CH}_{2}, \mathrm{C}-16\right), 55.4(\mathrm{CH}, \mathrm{C}-17), 11.7$ $\left(\mathrm{CH}_{3}, \mathrm{C}-18\right), 18.9\left(\mathrm{CH}_{3}, \mathrm{C}-19\right), 36.2(\mathrm{~d}, \mathrm{C}-20), 18.6\left(\mathrm{CH}_{3}, \mathrm{C}-21\right), 33.3$ $\left(\mathrm{CH}_{2}, \mathrm{C}-22\right), 25.4\left(\mathrm{CH}_{2}, \mathrm{C}-23\right), 45.1\left(\mathrm{CH}_{2}, \mathrm{C}-24\right), 28.7(\mathrm{CH}, \mathrm{C}-25), 19.1$ $\left(\mathrm{CH}_{3}, \mathrm{C}-26\right), 19.7\left(\mathrm{CH}_{3}, \mathrm{C}-27\right), 22.6\left(\mathrm{CH}_{2}, \mathrm{C}-28\right), 11.8\left(\mathrm{CH}_{3}, \mathrm{C}-29\right)$,
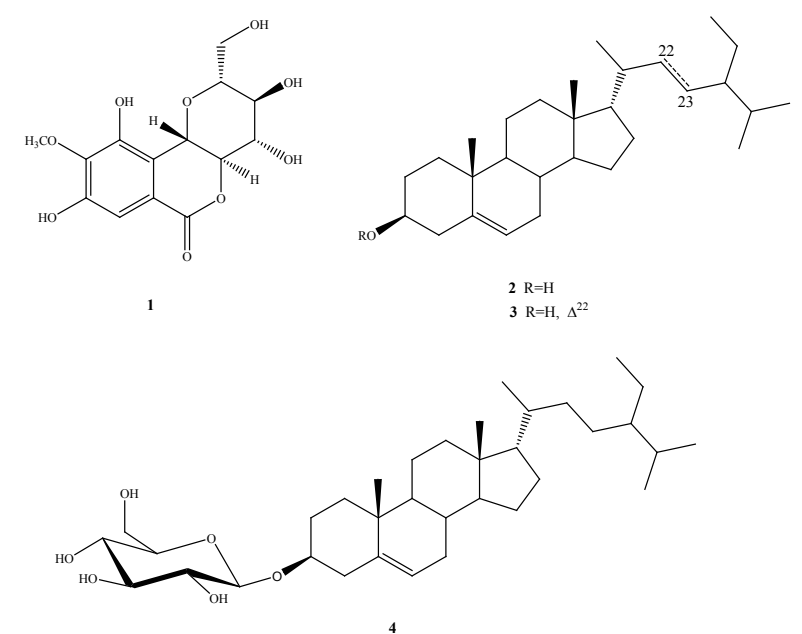

Figure 1: Chemical structures of compounds isolated from the roots of Cissus populnea. Bergenin (1); Mixture of $\beta$-sitosterol and stigmasterol $(2+3)$; Daucosterol (4). 
Citation: Nyemb JN, Djankou MT, Talla E, Tchinda AT, Ngoudjou DT, et al. (2018) Antimicrobial, a-Glucosidase and Alkaline Phosphatase Inhibitory Activities of Bergenin, The Major Constituent of Cissus populnea Roots. Med Chem (Los Angeles) 8: 021-025. doi: 10.4172/21610444.1000492

$100.8\left(\mathrm{CH}, \mathrm{C}-1^{\prime}\right), 73.5\left(\mathrm{CH}, \mathrm{C}-2^{\prime}\right), 76.8\left(\mathrm{CH}, \mathrm{C}-3^{\prime}\right), 70.1\left(\mathrm{CH}, \mathrm{C}-4^{\prime}\right), 76.7$ $\left(\mathrm{CH}, \mathrm{C}-5^{\prime}\right), 61.1\left(\mathrm{CH}_{2}, \mathrm{C}-6^{\prime}\right)$.

\section{In vitro antimicrobial assays}

Microorganisms and culture media: Eight microorganisms including four bacterial strains (Salmonella typhi ATCC6539, Salmonella typhimurium (isolate), Pseudomonas aeruginosa ATCC9721, Escherichia coli (isolate)) and four yeasts (Candida albicans ATCC9002, Candida parapsilosis ATCC22019, Candida krusei (isolate) and Candida albicans (isolate)), were used for the assays. E. coli was isolated from Ayub Theacthing Hospital of Abbottabad (Pakistan), S. typhimurium, C. krusei and C. albicans were isolates from the Laboratory of Bacteriology and Mycology of the "Centre Pasteur" of Yaounde, Cameroon while the reference strains were obtained from American Type Culture Collection (ATCC). Microorganisms were maintained on agar slant in refrigerator at $4^{\circ} \mathrm{C}$. Mueller Hinton Agar (MHA) was used for the activation of tested Gram-negative bacteria while Mueller Hinton Broth (MHB) was used for the in vitro antibacterial assay as culture media.

Antibacterial assay: The Minimal Inhibitory Concentrations and Minimal Bactericidal Concentrations (MICs and MBCs) of the samples and reference antibiotic were determined by the microdilution method using the MTT colorimetric assay as previously described by Nyemb et al. [27].

Anticandidal assay: For the anticandidal activity of all samples, the broth microdilution method previously described by Dzoyem et al. [32] was used for the determination of MICs and Minimum Fungicidal Concentrations (MFCs)

$\alpha$-Glucosidase inhibitory activity: The $\alpha$-glucosidase inhibition was evaluated according to the spectrophotometric method reported by Nyemb et al. [27], in which a-glucosidase from Saccharomyces cerevisiae (baker's yeast) was used. Baker's yeast is widely used for enzymatic assays due to its availability and ease of handling $[27,28,32,33]$.

Alkaline phosphatase inhibition assay: The luminescence method using CDP-Star as substrate for the determination of enzyme inhibition of samples on human tissue non-specific alkaline phosphatase ( $h$-TNAP) and human intestine alkaline phosphatase ( $h$-IAP) enzymes, was used as previously reported by Nyemb et al. [27].

\section{Results and Discussion}

\section{Compounds isolation}

The crude $\mathrm{MeOH}$ extract of $C$. populnea roots was separated by column chromatography of silica gel yielded four compounds reported for the first time from this species. The structure of all compounds was elucidated using ESI-TOF MS, NMR spectroscopy and by comparison with previous reported data of similar compounds. Thus, the isolated compounds (Figure 1) were identified as Bergenin (1) [22], a mixture (2.4:1) of $\beta$-sitosterol (2) and stigmasterol (3) [33,34] and Daucosterol ( $\beta$-sitosterol-3-O- $\beta$-D-glucoside, 4) [34].

\section{Antimicrobial activity}

All the isolated compounds were screened for their antimicrobial activity using the microbroth dilution method against eight microorganisms including four Gram (-) bacteria and four yeasts.

Regarding the results of the antibacterial activity (Table 1), it appears that Bergenin (1) was active against all the four selected bacteria including ATCC strains and clinical phenotypes, with MICs ranging from $8 \mu \mathrm{g} / \mathrm{mL}$ to $64 \mu \mathrm{g} / \mathrm{mL}$. Bergenin (1) exhibited a significant antibacterial activity against $P$. aeruginosa ATCC9721 $(8 \mu \mathrm{g} / \mathrm{mL})$, and moderate activities against the others bacterial strains $[35,36]$. The mechanism of antibiosis of Bergenin (1) determined by the MIC index (MBC/MIC values) suggested its bactericidal effect $(\mathrm{MBC} / \mathrm{MIC} \leq 4)$ on all the tested Gram-negative bacteria [36-38]. These results suggest that Bergenin (1) could be able to easily cross the complex and multilayered lipopolysaccharide cell walls of the tested Gram-negative bacterial strains [39]. The significant bactericidal activity $\left(\mathrm{MIC}<10 \mu \mathrm{g} \cdot \mathrm{mL}^{-1}\right.$ and $\mathrm{MBC} / \mathrm{MIC} \leq 4)$ of Bergenin (1) against $P$. aeruginosa recorded in this study is very important since $P$. aeruginosa is considered as an important nosocomial pathogen highly resistant to clinically used antibiotics, causing a wide spectrum of infections and leading to substantial morbidity and mortality [40]. However, the activity of Bergenin (1) against all the tested Gram-negative bacteria was lower comparing to the reference antibiotic compound Ciprofloxacine $(0.5$ $\left.\mu \mathrm{g} \cdot \mathrm{mL}^{-1}\right)$. Among the other compounds, only Daucosterol (4) exhibited a moderate activity $\left(32 \mu \mathrm{g} \cdot \mathrm{mL}^{-1}\right)$ against $E$. coli while its activity against P. aeruginosa was low $\left(128 \mu \mathrm{g} \cdot \mathrm{mL}^{-1}\right)[35,36]$.

No compound exhibited any antifungal activity (MIC $\left.>128 \mu \mathrm{g} \cdot \mathrm{mL}^{-1}\right)$ at the tested concentration against all the tested yeasts. This could probably explain why C. populnea is not used in traditional medicine to treat fungal infections. The inactivity of this compound towards all strain of tested yeast could be probably due to the peculiar structure of the external layers of yeasts, as for example C. albicans presents a thick cell wall where $\beta$-glucans and chitin are abundant [41].

Previously, antimicrobial studies were reported for Bergenin (1) [23], using agar diffusion method against standard and isolate microorganisms: E. coli (ATCC25922 and isolate), Salmonella enteritidis (ATCC11076 and isolate), P. aeruginosa (ATCC27853 and isolate), Enterococcus faecalis (ATCC49212), Staphylococcus aureus (ATCC49213), C. albicans (ATCC36232 and isolate), Candida guilliermondii (ATCC 6260 and isolate), Aspergillus flavus (ATCC32612 and isolate), Aspergillus nidulans (ATCC 28901 and isolate), Aspergillus niger (isolate), Candida tropicalis (isolate), Shigella sonnei (isolate), Serratia marcenses (isolate), Klebsiella pneumoniae (isolate), Enterococcus faecalis (isolate) and Staphylococcus aureus (isolate). Bergenin (1) was found to be inactive against all the tested Gram positive and Gram-negative bacteria, while it shown weak to moderate activities against Filamentous fungi and all yeasts [23]. $\beta$-sitosterol (2) formally isolated from Citrus grandis fruits, shown activities against gram-positive (Bacillus cereus, Bacillus subtilis and Staphylococcus aureus) and gram-negative (E. coli and Salmonella enteritidis) bacteria, with MIC value of $300 \mu \mathrm{g} / \mathrm{mL}$ [42]. Daucosterol (4) isolated from the leaves of Paulinna pinnata demonstrated antibacterial activity against $E$. coli and $P$. aeruginosa with MIC values of 25 and 100 $\mu \mathrm{g} / \mathrm{mL}$ respectively [43].

\section{Enzymatic activity}

The enzymatic activity of the isolated compounds was also evaluated in vitro against $\alpha$-glucosidase and alkaline phosphatase ( $h$-TNAP and $h$-IAP) (Table 2). All the tested compounds were weakly active against the $\alpha$-glucosidase enzyme at the tested concentration. The glucosidase activity recorded here for Bergenin (1) is not surprising since Kashima et al. [44] also reported its low inhibitory activity $(22.7 \pm 0.6 \%$ at 300 $\mu \mathrm{M})$ against the same $\alpha$-glucosidase enzyme from baker's yeast but at the concentration of $0.2 \mathrm{U} / \mathrm{mL}$. $\beta$-Sitosterol (2) and Daucosterol (4) also showed activities against yeast and bacterial $\alpha$-glucosidase enzyme $[34,45]$. However, the $\alpha$-glucosidase inhibitory activity of the same 
Citation: Nyemb JN, Djankou MT, Talla E, Tchinda AT, Ngoudjou DT, et al. (2018) Antimicrobial, a-Glucosidase and Alkaline Phosphatase Inhibitory Activities of Bergenin, The Major Constituent of Cissus populnea Roots. Med Chem (Los Angeles) 8: 021-025. doi: 10.4172/21610444.1000492

\begin{tabular}{|c|c|c|c|c|c|c|c|c|c|c|c|c|c|}
\hline \multirow{2}{*}{\multicolumn{2}{|c|}{ Microorganisms }} & \multicolumn{12}{|c|}{ Tested compounds, MICs, MBCs and MFC values $(\mu \mathrm{g} / \mathrm{mL})$} \\
\hline & & \multicolumn{3}{|c|}{1} & \multicolumn{3}{|c|}{$2+3$} & \multicolumn{4}{|c|}{4} & \multicolumn{2}{|c|}{ Standard drug } \\
\hline \multicolumn{2}{|l|}{ Bacterial strains } & MIC & MBC & & $\mathrm{x}$ & MIC & MBC & $\mathrm{X}$ & MIC & MBC & $\mathrm{X}$ & MIC & MBC \\
\hline \multirow{2}{*}{ E. coli } & Isolate & 64 & - & & 1 & - & - & 1 & 32 & 32 & 1 & 0.5 & 0.5 \\
\hline & ATCC6539 & 32 & & 28 & 4 & - & - & I & - & - & l & 0.5 & 1 \\
\hline S. typhi & Isolate & 64 & & 28 & 2 & - & - & 1 & - & - & 1 & 0.5 & 0.5 \\
\hline P. aeruginosa & ATCC9721 & 8 & & 32 & 4 & - & - & 1 & 128 & - & 1 & 0.5 & 1 \\
\hline \multicolumn{2}{|c|}{ Yeasts } & MIC & MFC & & $x$ & MIC & MFC & $\mathrm{x}$ & MIC & MFC & $\mathrm{x}$ & MIC & MFC \\
\hline \multirow{2}{*}{ C. albicans } & ATCC9002 & - & - & & I & - & - & 1 & - & - & I & 0.5 & 64 \\
\hline & Isolate & - & - & & 1 & - & - & 1 & - & - & 1 & 0.25 & 64 \\
\hline C. parapsilosis & ATCC22019 & - & - & & I & - & - & I & - & - & 1 & 2 & 16 \\
\hline C. krusei & Isolate & - & - & & 1 & - & - & 1 & - & - & 1 & 2 & 64 \\
\hline
\end{tabular}

(-): >128 $\mathrm{gg} / \mathrm{mL}$; /: Not determined; X: MBC(MFC)/MIC; Standard drug: Ciprofloxacin and Ketoconazole respectively for antibacterial and antifungal assays. E. coli; Escherichia coli; S. typhi: Salmonella typhimurium; P. aeruginosa; Pseudomonas aeruginosa; C. albicans: Candida albicans; C. parapsilosis; Candida parapsilosis; C. krusei: Candida krusei. In bold: significant activity [47].

Table 1: MICs, MBCs and MFCs $\left(\mu \mathrm{g} \cdot \mathrm{mL}^{-1}\right)$ of isolated compounds on tested microorganisms.

\begin{tabular}{|c|c|c|c|}
\hline \multirow{3}{*}{ Code } & \multirow{2}{*}{$\alpha$-Glucosidase } & \multicolumn{2}{|c|}{ Alkaline Phosphatase } \\
\hline & & $h$-TNAP & $h$-IAP \\
\hline & \multicolumn{3}{|c|}{$\mathrm{IC}_{50}(\mu \mathrm{M}) \pm \mathrm{SEM}$ or $\%$ Inhibition } \\
\hline 1 & $17.89 \%$ & $1.62 \pm 0.01$ & $12.86 \pm 0.03$ \\
\hline $2+3$ & $8.12 \%$ & 1 & I \\
\hline 4 & $28.32 \%$ & $48.24 \pm 1.02$ & $75.11 \pm 1.39$ \\
\hline Acarbose & $234.6 \pm 2.01$ & 1 & 1 \\
\hline Levamisole & 1 & $20.2 \pm 1.9$ & I \\
\hline L-Phenylalanine & l & 1 & $80.2 \pm 0.001$ \\
\hline
\end{tabular}

I: not determined; $I C_{50}$ is the concentration at which $50 \%$ of the enzyme activity is inhibited.

Table 2: $\alpha$-Glucosidase and alkaline phosphatase inhibition of the isolated compounds.

compound can be different in different reports, due to the fact that the enzyme inhibition is dependent on the concentration of the substrate, the concentration of the enzyme, the concentration and the $\mathrm{pH}$ of the buffer solution and the duration of incubation with the enzyme.

Bergenin (1) was active against the two alkaline phosphatase isoenzymes, $h$-TNAP and $h$-IAP used in this study. It exhibited high potency against $h$-TNAP and $h$-IAP with the $\mathrm{IC}_{50}$ values of $1.62 \pm$ $0.01 \mu \mathrm{M}$ and $12.86 \pm 0.03 \mu \mathrm{M}$, respectively. However, this activity of Bergenin (1) was $\sim 8$ fold more selective for $h$-TNAP over $h$-IAP and was respectively $\sim 12$ and $\sim 6$ fold higher compared to the positive controls levamisole and L-phenylalanine. Daucosterol (4) was also evaluated for its alkaline phosphatase inhibitory activities and exhibited good inhibitory activities against both isoenzymes but with a slight specificity for $h$-TNAP.

\section{Conclusion}

As far as we are aware, this is the first report regarding the isolation of compounds from the roots of C. populnea. This first phytochemical study of the roots of C. populnea led to the isolation of Bergenin (1) as the major compound along with Daucosterol (4) and a mixture (2:1) of $\beta$-Sitosterol and Stigmasterol. The alkaline phosphatase inhibitory activity of Bergenin (1) and Daucosterol (4) was also evaluated for the first time in this study and both compounds were found to have potent activities against the two isoenzymes TNAP and IAP used. With a broad spectrum of antimicrobial activity against the tested Gramnegative bacteria, Bergenin (1) might be the active constituent at least in part, responsible for the previous antibacterial activities observed for this plant. The results showed that $C$. populnea could be a good source of antibacterials and APs inhibitors.

\section{Acknowledgements}

JNN gratefully acknowledge UNESCO-TWAS and CIIT for the financial support to this project through the 2015 CIIT-TWAS Sandwich Postgraduate Fellowship (No. 3240287163). Authors are thankful to Mr. Victor NANA from the National Herbarium of Cameroon for identification of plants, and to Prof. Dr. Sophie Laurent and Dr. Celine Henoumont of the NMR and Molecular Imaging Laboratory, Department of General, Organic and Biomedical Chemistry, Faculty of Medicine and Pharmacy, University of Mons (Belgium), for their assistance in NMR and MS analysis of compounds. We also want to express our gratitude to the Medical Microbiology laboratory, Pasteur Centre, Yaoundé (Cameroon) and to the Ayub Theacthing Hospital of Abbottabad (Pakistan) for providing some clinical bacteria.

\section{References}

1. Soejima A, Wen J (2006) Phylogenetic analysis of the grape family (Vitaceae) based on three chloroplast markers. American Journal of Botany 93: 278-287.

2. Abu AH, Ofukwu RA, Mazawaje D (2009) A Study of traditional animal health care in Nasarawa State, Nigeria. American-Eurasian Journal of Sustainable Agriculture 3: 468-472.

3. Soladoye MO, Chukwuma EC (2012) Quantitative phytochemical properties of the leaves of Cissus populnea Guill \& Perr - an important medicinal plant in Nigeria. Archives of Applied Science Research 4: 200-206.

4. Soladoye MO, Chukwuma EC (2012) Phytochemical analysis of the stem and root of Cissus populnea (Vitaceae) - an important medicinal plant in Central Nigeria. Phytologia Balcanica 18: 149-153.

5. Shuaibu MN, Wuyep PA, Yanagi T, Hirayama K, Tanaka T, et al. (2008) The use of microfluorometric method for activity-guided isolation of antiplasmodial compound from plant extracts. Parasitology Research 102: 1119-1127.

6. Simo TR, Telefo BF, Nyemb JN, Yemele DM, Njina SN, et al. (2014) Anticancer and antioxidant activities of methanol extracts and fractions of some Cameroonian medicinal plants. Asian Pacific Journal of Tropical Medicine 7 : S442-S447.

7. Adebayo JO, Krettli AU (2011) Potential antimalarials from Nigerian plants: A review. Journal of Ethnopharmacology 133: 289-302. 
Citation: Nyemb JN, Djankou MT, Talla E, Tchinda AT, Ngoudjou DT, et al. (2018) Antimicrobial, a-Glucosidase and Alkaline Phosphatase Inhibitory Activities of Bergenin, The Major Constituent of Cissus populnea Roots. Med Chem (Los Angeles) 8: 021-025. doi: 10.4172/21610444.1000492

8. Azokou A, Kone MW, Koudou BG, Bi HFT (2013) Larvicidal potential of some plants from West Africa against Culex quinquefasciatus (Say) and Anopheles gambiae Giles (Diptera: Culicidae). Journal of Vector Borne Diseases 50: 103-110.

9. Kone WM, Atindehou KK, Traore D, Dosso M (2004) Traditional medicinal plants in north Cote-d'Ivoire: screening of 50 medicinal plants for antibacterial activity. Journal of Ethnopharmacology 93: 43-46.

10. Osibote EAS, Ogunlesi M, Okiei W, Asekun T, Familoni OB (2010) Assessment of antimicrobial activity of the essential oil from the stem powder of Cissus populnea and the leaves of Sesamum radiatum, herbal medications for male infertility factor. Research Journal of Medicinal Plants 4: 14-20.

11. Moody JO, Ojo OO, Omotade OO, Adeyamo AA, Olumeuse PE, et al. (2003) Anti-sickling potential of a Nigeria herbal formula (Ajawaron HF) and the major plant compound (Cissus populnea L. CPK). Phytotherapy Research 17: 11731176.

12. Ojekale AB, Lawal OA, Lasisi AK, Adeleke TI (2006) Phytochemisty and Spermato-genic Potentials of Aqueous Extract of Cissus populnea (Guill. and Per) Stem Bark. The Scientific World Journal 6: 2140-2146.

13. Geidam MA, Adoga GI, Sanda FA (2004) Effects of aqueous stem bark extract of Cissus populnea on some serum enzymes in normal and Alloxan induced diabetic rats. Pakistan Journal of Biological Sciences 7: 1427-1429.

14. Osibote E, Noah N, Sadik O, McGee D, Ogunlesi M (2011) Electrochemical sensors, MTT and immunofluorescence assays for monitoring the proliferation effects of Cissus populnea extracts on Sertoli cells. Reproductive Biology and Endocrinology 9: 1-14.

15. Ibrahim H, Mdau BB, Ahmed A, llyas M (2011) Anthraquinones of Cissus populnea Guill \& Perr (amplidaceae). African Journal of Traditional, Complementary and Alternative Medicines 8: 140-143.

16. Ibrahim H, Rai PP, Bangudu AB (1993) Pharmacognostic studies of the stem bark of Cissus populnea Guill \& Perr. Glimpses in Plant Research 1: 175-180.

17. Chi CY, Wang F, Lei T, Xu SY, Hong AH, et al. (2010) Studies on the chemica constituents from Cissus pteroclada. Journal of Chinese Medicinal Materials 33: 1566-1568.

18. Lin CF, Hwang TL, Lin SY, Huang YL (2012) Bioactive isocoumarins from Cissus pteroclada. Journal of Chinese Medicine 23: 41-49.

19. Khan H, Amin H, Ullah A, Saba S, Rafique J, et al. (2016) Antioxidant and Antiplasmodial Activities of Bergenin and 11-O-Galloylbergenin Isolated from Mallotus philippensis. Oxidative Medicine and Cellular Longevity 2016: 1-6.

20. Nunomura RCS, Oliveira VG, Da Silva SL, Nunomura SM (2009) Characterization of Bergenin in Endopleura uchi Bark and its Anti-Inflammatory Activity. Journal of the Brazilian Chemical Society 20: 1060-1064.

21. Subramanian $R$, Subbramaniyan $P$, Raj $V$ (2015) Isolation of Bergenin from Peltophorum pterocarpum flowers and its bioactivity. BENI-SUEF University Journal of Basic and Applied Sciences 4: 256-261.

22. Caldas CS, De Simone CA, Pereira MA, Malta VRS, Carvalho RLP, et al (2002) Bergenin monohydrate, a constituent of Hurmiria balsamifera, at $120 \mathrm{~K}$ Acta Crystallographica Section E 58: 609-611.

23. Da Silva SL, De Oliveira VG, Yano T, Nunomura RCS (2009) Antimicrobia activity of Bergenin from Endopleura uchi (Huber) Cuatrec Acta amazonica 39: 187-192.

24. Maxwell DB, Fisher EA, Ross-Clunis HA, Estep HL (1986) Serum alkaline phosphatase in diabetes mellitus. Journal of the American College of Nutrition 5: 55-59.

25. Playford RJ, Pither CR, Gao R, Middleton SJ (2013) Use of the a-glucosidase inhibitor acarbose in patients with 'Middleton syndrome': normal gastric anatomy but with accelerated gastric emptying causing postprandial reactive hypoglycemia and diarrhea. Canadian Journal of Gastroenterology 27: 403-404.

26. Zhang BB, Moller DE (2000) New approaches in the treatment of type 2 diabetes. Current Opinion in Chemical Biology 4: 461-467.

27. Nyemb JN, Tchinda TA, Talla E, Nanga EB, Ngoudjou TD, et al. (2018) Vitellaroside, a new cerebroside from Vitellaria paradoxa (Sapotaceae) and its bioactivities. Natural Products Chemistry \& Research (In press).

28. Hashmi MA, Shah HS, Khan A, Farooq U, Iqbal J, et al. (2015) Anticancer and Alkaline Phosphatase Inhibitory Effects of Compounds Isolated from the Leaves of Olea ferruginea Royle. Records of Natural Products 9: 164-168.
29. Kazmi M, Zaib S, Amjad ST, Khan I, Ibrar A, et al. (2017) Exploration of aroyl/heteroaroyl iminothiazolines featuring 2,4,5-trichlorophenyl moiety as a new class of potent, selective, and in vitro efficacious glucosidase inhibitors. Bioorganic Chemistry 74: 134-144.

30. Park H, Hwang KY, Oh KH, Kim YH, Lee JY, et al. (2008) Discovery of nove a-glucosidase inhibitors based on the virtual screening with the homologymodeled protein structure. Bioorganic and Medicinal Chemistry 16: 284-492.

31. Choi CW, Choi YH, Cha MR, Yoo DS, Kim YS, et al. (2010) Yeast a-glucosidase inhibition by isoflavones from plants of Leguminosae as an in vitro alternative to acarbose. Journal of Agricultural and Food Chemistry 58: 9988-9993.

32. Dzoyem JP, Tchuenguem TR, Kuiate JR, Teke NG, Kechia FA, et al. (2014) In Vitro and in vivo antifungal activities of selected Cameroonian dietary spices. BMC Complementary and Alternative Medicine 14: 1-8.

33. De-Eknamkul W, Potduang B (2003) Biosynthesis of beta-sitosterol and stigmasterol in Croton sublyratus proceeds via a mixed origin of isoprene units. Phytochemistry 62: 389-398.

34. Sheng Z, Dai H, Pan S, Wang H, Hu Y, et al. (2014) Isolation and Characterization of an a-Glucosidase Inhibitor from Musa spp. (Baxijiao) Flowers. Molecules 19: 10563-10573.

35. Kuete V (2010) Potential of Cameroonian plants and derived-products against microbial infections: a review. Planta Medica 76: 1-13.

36. Talla E, Yaya GAJ, Mokale KAL, Abdou JP, Tchinda A, et al. (2016) Chemica constituents from Erythrina droogmansiana (Fabaceae), radical scavenging and antibacterial potential of some extracts and compounds. Natural Products: An Indian Journal 12: 012-020.

37. Mbaveng AT, Kuete V, Mapunya BM, Beng VP, Nkengfack AE, et al. (2011) Evaluation of four Cameroonian medicinal plants for anticancer, antigonorrheal and antireverse transcriptase activities. Environmental Toxicology and Pharmacology 32: 162-167.

38. Mbaveng AT, Ngameni B, Kuete V, Simo IK, Ambassa P, et al. (2008) Antimicrobial activity of the crude extracts and five flavonoids from the twigs of Dorstenia barteri (Moraceae). Journal of Ethnopharmacology 116: 483-489.

39. Rakholiya K, Vaghela P, Rathod T, Chanda S (2014) Comparative Study of Hydroalcoholic Extracts of Momordica charantia L. against Foodborne Pathogens. Indian Journal of Pharmaceutical Sciences 76: 148-156.

40. Cardoso O, Alves AF, Leitao R (2007) Surveillance of antimicrobial susceptibility of Pseudomonas aeruginosa clinical isolates from a central hospital in Portugal. Journal of Antimicrobial Chemotherapy 60: 452-454.

41. Bisio A, Schito AM, Ebrahimi SN, Hamburger M, Mele G, et al. (2015) Antibacterial compounds from Salvia adenophora Fernald (Lamiaceae). Phytochemistry 110: 120-132.

42. Matook SM, Fumio H (2005) Evaluation of the antimicrobial activity of extract from limitan (Citrus grandis Osbeck) fruit peel. Pakistan Journal of Biological Sciences 8: 1090-1095.

43. Lunga PK, Qin XJ, Yang XW, Kuiate JR, Du ZZ, et al. (2014) Antimicrobial steroidal saponin and oleanane-type triterpenoid saponins from Paullinia pinnata. BMC Complementary and Alternative Medicine 14: 1-7.

44. Kashima Y, Yamaki H, Suzuki T, Miyazawa M (2013) Structure-activity relationships of bergenin derivatives effect on $\alpha$-glucosidase inhibition. Journal of Enzyme Inhibition and Medicinal Chemistry 28: 1162-1170.

45. Tabussum A, Riaz N, Saleem M, Ashraf M, Ahmad M, et al. (2013) $\alpha$-Glucosidase inhibitory constituents from Chrozophora plicata. Phytochemistry Letters 6 : 614-619. 